\title{
Application of Stitching Design in Fashion Design
}

\author{
Shuli Wen ${ }^{1}$
}

${ }^{1}$ Jiangxi Institute of Fashion Technology, Nanchang Jiangxi, China, 330201

KEYWORDS: Stitching Design, Fashion Design, Fabrics, Color, Structure

\begin{abstract}
People clothing is constantly changing with the times, including clothing styles, fabrics, colors and so on. Costume designer to meet the new demands of modern people, their design has also done a lot of bold innovation and exploration. Stitching design as an important part of fashion design, fashion designer in modern stitching design also made a bold improvement to meet the people's existing garment diversified requirements. In this paper, the basic concept of stitching design made related presentations, focusing on analysis of the design in the stitching fabric stitching, stitching color and style stitching and other related use.
\end{abstract}

\section{Introduction}

Modern technology in the traditional splicing technology improvement and innovation, mosaic design is one of the important modern costume design expression, it is primarily for assembly by a combination of different fabrics, color, texture, and thus bring different visual, tactile experience, bring people freedom, comfort, personal psychological experience to meet the diverse requirements of modern clothing. It is now increasingly garment stitching welcomed by the people, costume design by now becoming a relatively large number of practices, especially in spring and summer clothing, the majority of consumers. In today's conference, major clothing and apparel brands, stitching become a major source of inspiration for fashion designers important, more and more outstanding works stitching garments presented in front of everyone.

\section{Related Overview of Design}

Splicing originated in the Middle Ages, has been for thousands of years, at the beginning of stitching used in clothing design is mainly to make people more comfortable to wear. With the development of modern fashion, stitching design in the pursuit of comfort is based on the increase of freedom, personality and other spiritual elements. Mosaic Art in the United States formally proposed the 18th century, when the clothing splicing technology is mainly used in the textile color printing, after gradually be extended to the fabric. In the nineteenth century, the US garment industry rapid development, coupled with the rapid development of American society to pursue personality, fashion, garment stitching Art. Mosaic Art not only meet the people's pursuit of diversification clothing requirements, but also saves the cost of production, highlighting the personality of fashion design, fashion design show the creativity and artistry. With the rapid development of the apparel industry to splice design in the 21st century is also very different from the original traditional design, clothing splicing technology has risen to an art behavior. Clothing is an important to express their attitude to life in the form of clothing splicing technology to meet the people to improve existing clothing requirement. 
Clothing splicing factors influencing art at different times are different, each period has its own main factors, but the economy and culture of these two factors is the most important factor.

Mosaic art of clothing and economic factors are inextricably linked. Clothing splicing technology originated in the poverty class, the class of people due to economic conditions, they have a variety of different fabrics and colors of cloth stitched together, so as to ensure the integrity of the garment. After the rapid economic development and people's aesthetic changes, so that originally belonged to the poor class clothing art by more people welcome.

Culture is another of the important factors affecting the development of art stitching clothing. Pop Art in the contemporary art of stitching garments maximum. Pop Art originated in the United States and Europe, it is an important and unique art form, from the pursuit of freedom and constraint is the traditional art forms of the most important features. By design, not a pure pop style consistency of style, but a variety of styles mixed. It is the pursuit of popular, popular taste, against modernism pretentious GAO. He stressed the novelty and unusual and bold use of gaudy colors, giving a fresh feeling in the design. Pop Art has broken the traditional aesthetic concepts, under the influence of the arts, fashion design, many models have undergone tremendous changes, new elements appear more clothing in fashion design, consumers have gradually accepted and loved on this new style of clothing.

\section{Mosaic Design in Fashion Design}

Fabric is one of the three elements of clothing, it can be well reflected the style and characteristics of garments and clothing can enhance functional and aesthetic. Different fabrics have different flexibility, hardness, garment design process to select a suitable fabric for use in the corresponding parts, to meet the needs of the various parts of the body. Such as cuffs, elbows and other frequent friction and easily dirty parts should be selected wear dark fabrics. To increase the beauty of the clothing in the costume design process, in the back of the garment, the hem, the shoulder can be adapted to add soft fabrics in hard fabric to make clothes more elegant and gentle. Contrast can highlight a variety of fabrics fabric characteristics varied.

Splicing of different fabrics include knitted fabrics and woven fabric stitching, needle, woven fabrics and furs stitching. Knitted fabric has good elasticity and large elongation characteristics, particularly suitable exaggerated, loose clothing design. High production efficiency knitted fabric, and the fabric itself is personal, delicate, soft, full compliance with the requirements of modern clothes. However, because of their knitted fabrics knitted structure (coil structure), easy curling, retraction, the deformation, so have a great impact on the quality of clothes and the use of time. Woven fabric having a high strength, washable, wear-resistant properties, appropriate splicing of some woven fabrics in knitted fabric, easily deformable parts, especially in the collar, sleeves and the like. Fur splicing can make clothes more prominent personality and style of the clothes, the clothes also allow more wealth and fashion, highlighting the wearer confidence and avant-garde. In the costume design process, needles, woven fabrics appropriately added fur, can organically knitted fabrics and delicate and soft fur of wild, uninhibited organically combined to make it more sexy clothes.

Stitching the same fabric refers to different colors of stitching the same fabrics and stitching fabric opposite surface. Stitching the same fabric can effectively save the costume design and production time, greatly improve efficiency, but also to break the limitations of existing clothing styles, adding new elements of clothing design, sympathetic to bring a different visual effects, to meet clothes pursue diversified and personalized requirements of consumers. 
Color is the most important elements of costume design. People feel about the first piece of clothing from the color of the garment. Costume design by the depth of color, layers, arranged to form a garment unique beauty. Clothing color stitching may also be used in different parts of the body, such as the need to highlight parts of the body lines, the color should be used relatively deep color. Now currently in the process of costume design in the same color stitching the most common color mosaic method, adjacent color stitching and contrasting color stitching.

Near the same color stitching and stitching color refers to the use of the same color and the same color of light and dark shades in clothes design scattered arrangement for splicing. Since the color is similar to the neighboring color, the clothes will not feel much change unobtrusive, it brings a sense of serenity and peace. As in dark blue jeans, the pockets and cuffs, etc. You can use light blue, make clothes more individuality, let clothes rigid, and enhance the sense of active strength of the garment. Stitching the same color and neighboring colors are also easy to make clothes appear dull, to break the detention, the stitching is mainly used in the clothing of different fabrics. Different fabrics have different characteristics, color and fabric to make clothes with a design full of changes, highlighting the luxurious clothes, introverted, reserved.

Contrasting color stitching is to take color with distinctive color contrast stitching. At present, many fashion designers as bold breakthrough security mediocre design uses high brightness and color saturation. Such a strong color contrast make consumers refreshing, it brings a cheerful feeling, strongly appealing to the eye. This contrast color stitching garments particularly is suitable for some, such as the stage, party, queuing and other places. However, contrasting color stitching clothing color stitching the most difficult to grasp, the key must handle a variety of colors mosaic proportions make clothes color colorful and harmony.

Clothing structural stitching is also an important element in fashion design. It mainly includes non-separable structure stitching, detachable structure stitching, irregular stitching structure categories. Costume design to reflect the different clothing styles, different combinations of fabrics and colors, clothing architecture optimization, inseparable structure style stitching to make more innovative. As sweater collar, cuffs, hem stitching shirt fabric, so you can make clothes showing a two-piece style, reflecting a casual, stylish design, this design students and white-collar workers was generally welcome. Separate mosaic structure mainly refers to certain parts of clothes set some removable panels, such as the zipper, dark button, lining and so on. Such as coats generally better than warm down jacket, some consumers do not want to wear too bloated, increased coat lining can be the perfect solution, but also can show elegant maintain a perfect body lines. Irregular mosaic structure designed in the style, fabric, color and other aspects of the law are not clear, but this is mainly stitching techniques with theatrical performances that focus on fashion and aesthetic clothes and clothes do not need to consider the practicality of the situation .

\section{Conclusion}

Stitching design is important in modern fashion design technique of expression, in addition to fabric, color, mosaic structure, including the design, style stitching. Splicing technology to break the traditional clothing design, highlighting the clothing fashion sense and personality fit the needs of the consumers, so splicing technology has great room for development.

\section{References}

[1] Ke Yudan. mosaic art in the costume design application [J]. Grand Art, 2014, (11): 119.

[2] Yuan Bo. mosaic application in fashion design [J]. Popular Literature, 2014, (14): 114. 
[3] Chen Yajing. mosaic art in the costume design application [J]. fine arts, 2015, (8): 71-71.

[4] Yang Haijie. stitching applications in Fashion Design [J]. City tutor (the first half), 2015, (2): 196-196. 